\title{
Validation of MODIS and AVHRR sea surface temperature using bulk measurements
}

\author{
F. Eugenio ${ }^{* a}$, J. Marcello and E. Rovaris ${ }^{\mathrm{a}}$ \\ ${ }^{a}$ Signals and Communications Department. University of Las Palmas of G.C. \\ Campus Universitario de Tafira. Edificio de Electrónica y Telecomunicación. \\ 35017 Las Palmas de G.C., Spain
}

\begin{abstract}
The development of algorithms for the production of reliable Sea Surface Temperature (SST) data sets from space borne infrared radiometers has been pursued by different agencies since late 1960's. The current state of the art in SST retrieval from space is limited by radiometer window placement, radiometer noise, quality of pre-launch characterization, in-flight calibration quality, viewing geometry and, mainly, atmospheric correction. The correction to eliminate the atmospheric effects is a critical and complicated step in the validation process, because although the satellite observations are approaching sufficient quality for routine use, unfortunately the thermal structure in the upper $10 \mathrm{~m}$ of the ocean is complex and highly variable, so SST may be significantly different depending on the vertical depth of the in situ measurement, the local time of day, local conditions at the air-sea interface and the instrument used; so in order to properly merge skin and bulk temperatures, daytime match-ups have been excluded from the validation process. In this context, the validation of the NOAA-AVHRR/3 and TERRA-MODIS atmospheric correction algorithms for the retrieval of sea surface temperature from the Canary Islands-Azores-Gibraltar area is performed by using more than 1500 in situ temperature measurements, derived from the ARGO Data Collection System and by the Oceanographic Service of the University of Las Palmas Gran Canaria, over the period from December 2000 to September 2003.
\end{abstract}

Keywords: Remote sensing, oceanography, sea surface temperature, AVHRR, MODIS, bulk temperature, skin temperature.

\section{INTRODUCTION}

The sea surface temperature (SST) is an important geophysical parameter essential for quantitative studies of the Earth's atmosphere and oceans, being recognized as one of the main controllers and indicators of climate variability. SST measurements also have a wide variety of applications, including physical oceanographic research, support of commercial fishing activities, derivation of ocean feature charts and ocean surface current measurements useful in support of ship routing and pollution monitoring. Most satellite SST applications require accuracies around $0.5{ }^{\circ} \mathrm{C}$, with high spatial resolution $(0.5-10 \mathrm{~km})$ and temporal resolution of 2-4 observations per day [1].

Great advance in the measurement of SST was done with the launch of the five-channel Advanced Very High Resolution Radiometer (AVHRR/2) instruments aboard National Oceanic and Atmospheric Administration (NOAA) meteorological satellite (NOAA-7 (1981) to NOAA-14) included the so-called 'split-window' channels at 10.7 y $11.9 \mu \mathrm{m}$, allowing SST determination during the day time. The AVHRR/2 instruments, and nowadays the six-channel AVHRR/3 scanning radiometer, aboard NOAA-15, 16 \& 17 satellite are widely used to obtain SST. This is because: (i) AVHRR data can be acquired and processed in real time with a polar satellite ground receiving station of relatively low cost and with the corresponding ad-hoc processing tools, (ii) the low noise levels, expressed as a noise equivalent temperature difference ( $\mathrm{NE} \Delta \mathrm{T}<0.12{ }^{\circ} \mathrm{C}$ ), in the split-windows channels and, (iii) the appropriate in-flight non-linear calibration system of the thermal infrared channels.

* feugenio@dsc.ulpgc.es; phone 34928 452979; fax 34928451243 
In practice, the development of split-window algorithms is based on the brightness temperatures measured in the 10.311.3 $\mu \mathrm{m}$ and 11.5-12.5 $\mu \mathrm{m}$ bands (AVHRR channels 4 and 5) separately, and on the hypothesis that the sea surface temperature can be obtained by the following expression,

$$
T_{S}=\alpha T_{11}+\gamma\left(T_{11}-T_{12}\right)+\beta
$$

with, $T_{S}$ : Sea surface temperature; $T_{11}, T_{12}$ : AVHRR brightness temperature of channels 4 and 5 and $\alpha, \gamma, \beta:$ Constants coefficients.

The correction to eliminate the atmospheric effects is a critical and complicated step in the validation process because although the satellite observations are approaching sufficient quality for routine use, unfortunately the thermal structure in the upper $10 \mathrm{~m}$ of the ocean can be complex and highly variable, so SST may be significantly different depending on the vertical depth of the in-situ measurement, the local time of day, local conditions at the air-sea interface (wind speed) and the instrument used. All these variables complicate the validation process to obtain high quality products. Recent studies [2] indicate that in-situ (bulk) and satellite (skin) temperatures can only be merged (with an accuracy at the level of $0.1 \mathrm{~K}$ ) at night or when wind speed exceeds $6 \mathrm{~m} / \mathrm{s}$, however with low wind speeds and during daytime the only reliable measurement of the skin temperature is provided by high precision in-situ infrared radiometers.

In summary, for the validation of satellite retrieved SST, there are two classes of measurements: comparison of satellite data with in-situ bulk data and the use of precise radiometers to make measurements of surface skin temperature. Although the first technique is not suitable to establish the ultimate accuracy, it is essential to derive first accurate estimates of the SST and the achieved precision is more than enough for some applications.

The essential objective in this work is to obtain the error statistics of the SST algorithms used for AVHRR/3 and MODIS (Moderate Imaging Spectroradiometer) sensors when applied within our oceanographic area of interest.

\section{SATELLITE - IN SITU DATA COMPILATION}

The systematic procedure performed to obtain the matchup database between in situ SST measurements and nearlycoincident satellite observations is shown in Fig. 1. This procedure includes three steps, which will be explained next.

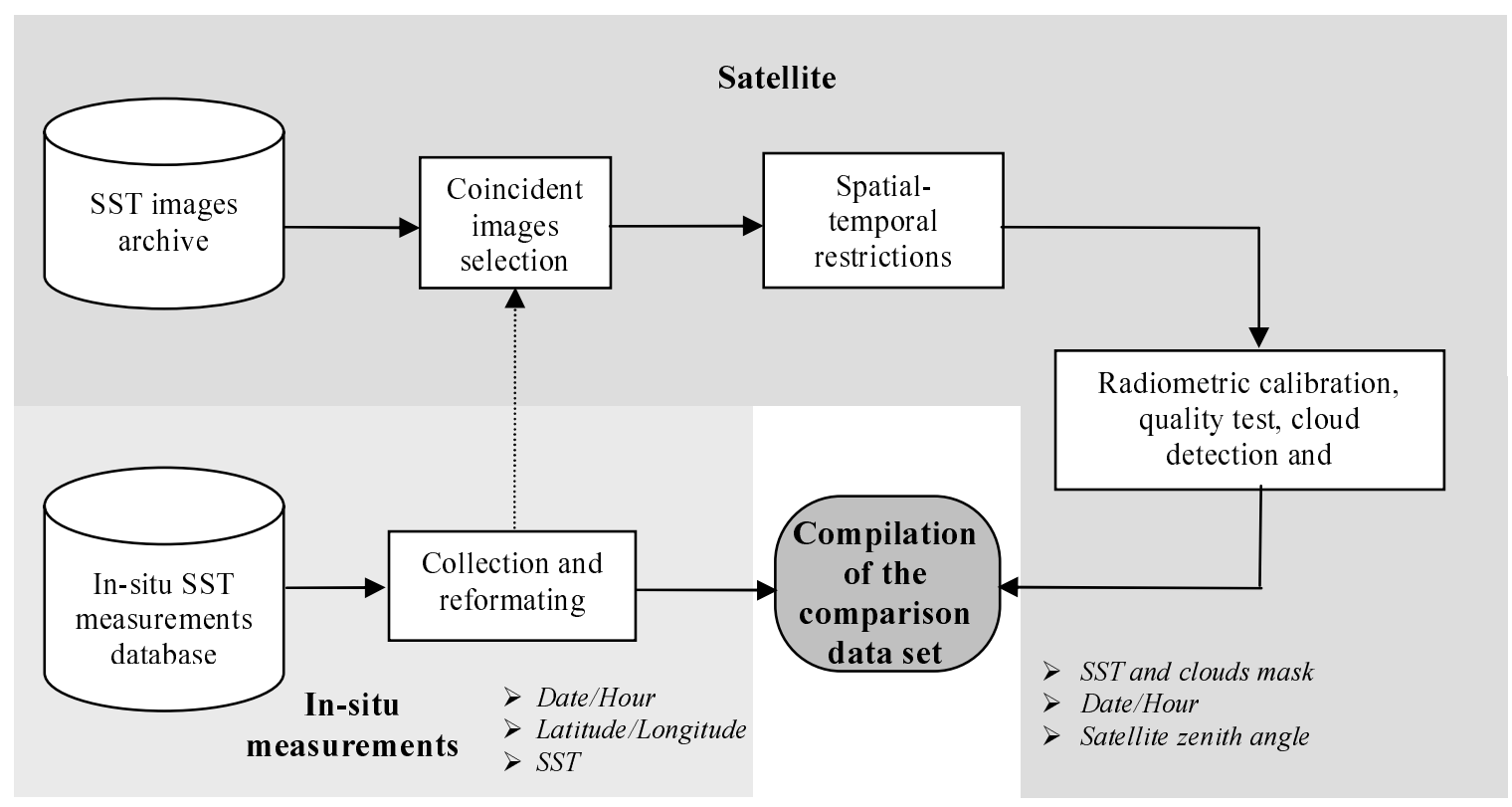

Fig. 1. Systematic procedure to generate the matchup data set. 


\subsection{In situ measurements}

The first step is the compilation of in situ sea surface temperature from different sources. In order to achieve a representative data set, observations should be selected with an adequate space-time distribution within the oceanographic region of interest, as well as having different observations conditions (i.e., different vision geometries).

Sea Surface Temperature measurements have been obtained during the time period comprised from December 2000 to September 2003. The measurements have been performed in the Canary Islands-Azores-Gibraltar (CANIGO) and the data was provided by profilers from the Argo Data Collection System [3] and the CTD measurements performed in two oceanographic transects by the Oceanographic Service of the University of Las Palmas Gran Canaria. In both cases the depth of the measurement was variable and not exceeding 10 meters. The number of available measurements was 1566 and its location is presented in Fig. 2.

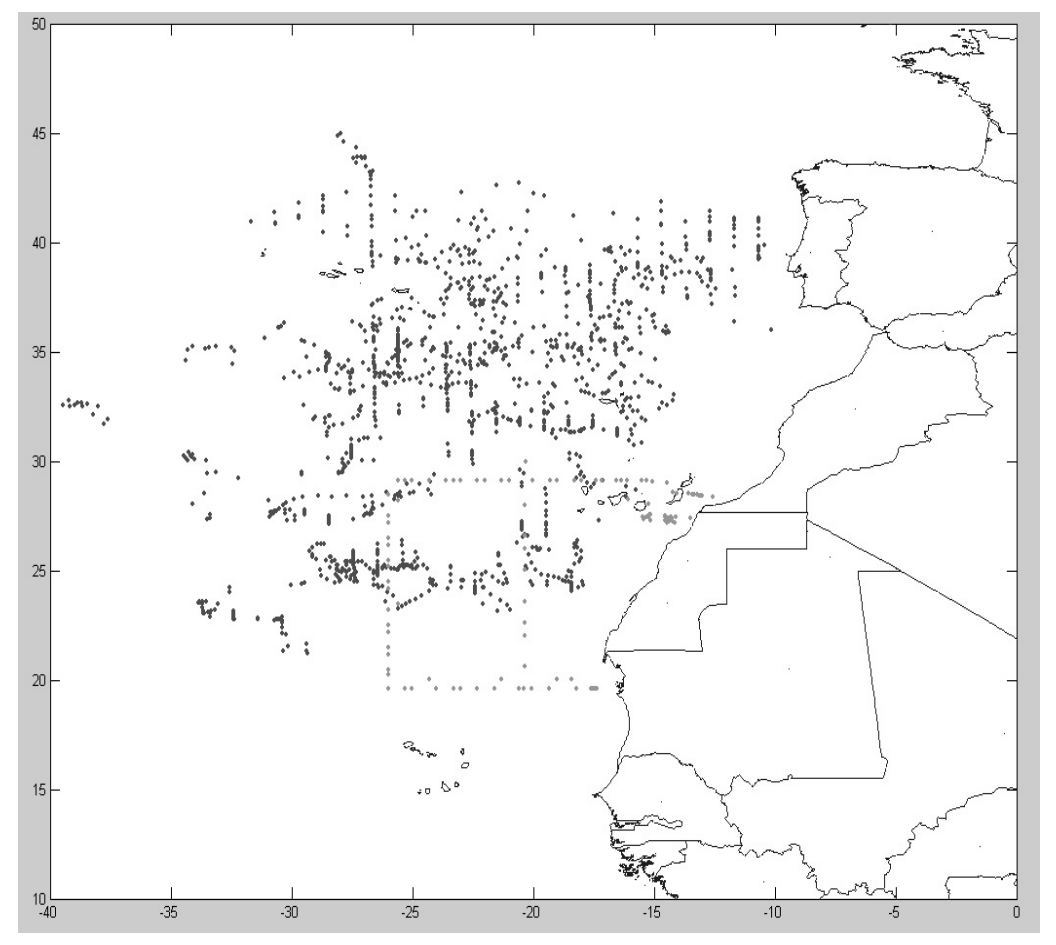

Fig. 2. Spatial distribution of the in-situ measurements (•) Oceanographic Service (ULPGC) and (•) ARGO Project.

\subsection{Satellite sensor data}

The AVHRR-3 NOAA 16 data are obtained in real-time from the satellite HRPT ground station at the Remote Sensing Center (Universidad de Las Palmas de Gran Canaria) [4]. Next, the corresponding processing stages are performed, mainly:

- $\quad$ Raw to NOAA level 1B conversion.

- Radiometric calibration.

- Cloud masks generation using the multi-band threshold method proposed by Saunders [5] and adapted to the region of analysis.

- Atmospheric correction to obtain SST maps, and 
- A two-stage procedure for an automatic image geometric correction using an orbital prediction model, to provide first-guess earth locations, followed by a global contour matching technique [6]. The approach aims at exploiting the maximum reliable information in the image to guide the matching algorithm. The following steps summarize the automatic global contour matching procedure: (1) estimation of the gradient energy map (edges) of the sensed image and detection of the cloudless (reliable) areas; (2) initialization of the contours positions, obtained after processing the geographical data available at the CIA World Data Base II; (3) obtaining of the transformation parameters (affine model) by means of a global contour optimization approach. Several experimental results have verified the robustness and accuracy of the proposed algorithms, demonstrating their capability of georeferencing satellite images within an error of one pixel.

The MODIS products are ordered at the NASA Distributed Active Archive Center, DAAC [7], and for the validation, the products required are the SST (MOD28L2), the Geolocation data set (MOD03) and the cloud mask (MOD35). Fig. 3 shows an example of SST maps retrieved for both sensors.

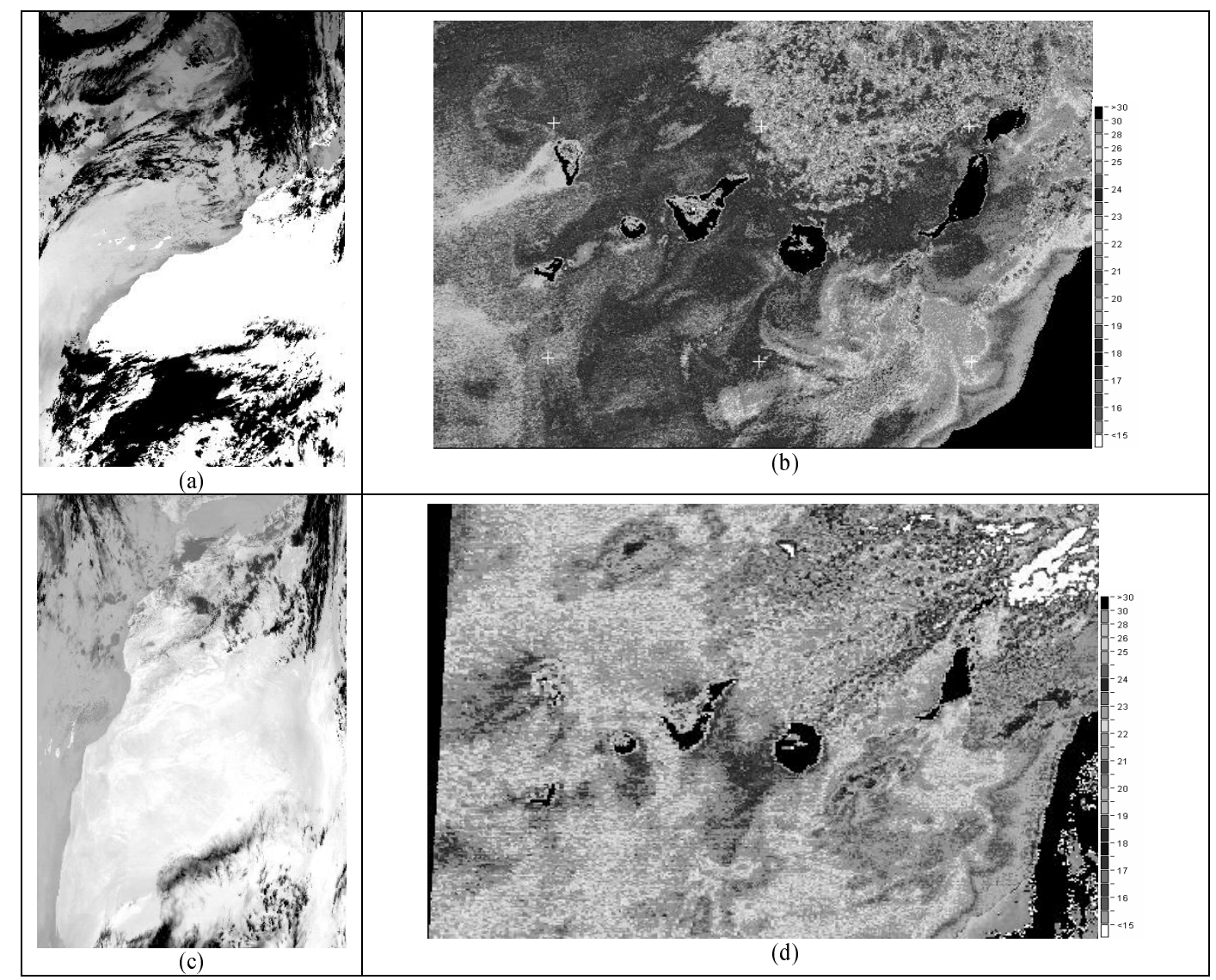

Fig. 3. SST images of $31^{\text {st }}$ October 2002. (a) AVHRR/3 (NOAA16) at 14:20 hr, (b) MODIS (TERRA) at 11:15 hr, (c) and (d) colour images of the Canary islands geometrically corrected and georeferenced to UTM projection from (a) and (b) respectively.

Temporal restrictions are applied, allowing a maximum temporal separation between satellite and in-situ data of \pm 30 minutes for day-time images, and \pm 1 hour for the night-time ones. This restrictive temporal filtering lead to an exclusion of around $40 \%$ of in situ measurements originally accounted for. Those temporal restrictions preserve the invariability of the oceanic conditions for both sets of measurements. 


\subsection{The compilation of comparison data sets}

To generate the matchup database several qualitative and quantitative tests are performed between in situ measurements and coincident satellite observations.

In the qualitative test, the geometrically corrected clouds mask is used to mask those pixels in the SST $5 \times 5$ sub-matrices contaminated by clouds. In the quantitative test we calculate the standard deviation of the SST in a window of $3 \times 3$ pixels centred in each position of the in situ measurement. If the standard deviation is less than $0.12{ }^{\circ} \mathrm{C}$ (sensor $\mathrm{NE} \Delta \mathrm{T}$ ), we choose the average values of the SST within the window as the corresponding SST of the in situ measurement. If the standard deviation is larger than $0.12{ }^{\circ} \mathrm{C}$ we analyse the other 8 windows of $3 \times 3$ inside the searching area of $5 \times 5$ pixels. That window having the minimum standard deviation not exceeding $0.12^{\circ} \mathrm{C}$ is used to retrieve the sea surface temperature.

The selected data sets, after passing all previous tests, are compiled to generate a comparison database. Table 1 provides a description of the different fields included in the generated database.

Table 1. Fields description and generated matchup data sets.

\begin{tabular}{|c|c|c|c|c|c|}
\hline \multicolumn{3}{|c|}{ SST in-situ data } & \multicolumn{3}{c|}{ Satellite sensor data } \\
\hline \hline Field & Parameter & Units & Field & Parameter & Units \\
\hline$\# 1$ & Date & ddmmyy & $\# 6$ & Date image & ddmmyy \\
\hline$\# 2$ & Hour & hours & $\# 7$ & $1^{\text {st }}$ line hour & hours \\
$\# 3$ & Latitude & degrees & $\# 8$ & Sea Surface Temperature & ${ }^{\circ} \mathrm{C}$ \\
\hline$\# 4$ & Longitude & degrees & $\# 9$ & Zenith angle secant $(\sec \theta)$ & - \\
\hline$\# 5$ & Temperature & ${ }^{\circ} \mathrm{C}$ & $\# 10$ & & \\
\hline$\ldots$
\end{tabular}

\section{AVHRR/3 ALGORITHM VALIDATION}

AVHRR flown on the NOAA series of polar orbiting satellites have provided SST measurements for over two decades. Different linear split-window algorithms have been implemented using in-situ ocean temperature measurements acquired around the world. However, the atmospheric dependence of the split-window coefficients, as analysed by Coll et al. [8] , does not recommend the use of global algorithms in areas having particular climatic conditions. It is not advisable, thus, the use of linear global algorithms with fixed-coefficients in the CANIGO area. So, after a thorough validation process, Eugenio [9] developed an operational split-window algorithm to perform the atmospheric correction of AVHRR/2 (NOAA-14) images for all zenith angles, valid for our zone of oceanographic interest and for any period of the year. The final expression has the following form:

$$
\begin{gathered}
S S T=a_{0} \cdot T_{4}+\left[a_{1}+a_{2} \cdot\left(T_{4}-T_{5}\right)\right]\left(T_{4}-T_{5}\right)+ \\
{\left[a_{3}+a_{4} \cdot\left(T_{4}-T_{5}\right)\right](\sec \theta-1)+a_{5}}
\end{gathered}
$$

where $T_{4}$ and $T_{5}$ are the brightness temperatures measured in channel 4 (10.3 to $\left.11.3 \mu \mathrm{m}\right)$ and channel 5 (11.5 to 12.5 $\mu \mathrm{m})$ and the coefficients, obtained by linear multidimensional regression analysis, are: $a_{0}=1.0344 ; a_{1}=2.0193 ; a_{2}=-$ $0.0921^{\circ} \mathrm{C}^{-1} ; a_{3}=1.5472{ }^{\circ} \mathrm{C} ; a_{4}=0.1565$ and $a_{5}=-0.6514{ }^{\circ} \mathrm{C}$. 
The validation of this algorithm for AVHRR/3 (NOAA 16) images, in our region of interest, was performed using a total of 100 match-ups (80 in night-time and the rest during the day) that passed all the restrictions mentioned in section II. The results are summarized at Table 2 and Fig. 4. The table also includes the evaluation of the algorithm accuracy for the night-time match-ups in order to achieve a consistent comparison taking into account the mentioned discrepancies between skin and bulk temperatures under daytime conditions.

Table 2. SST Error Statistics applying Eugenio Algorithm to the NOAA-16 AVHRR/3 Images.

\begin{tabular}{|c|l|c|c|c|}
\hline \hline \multirow{2}{*}{ SST estimation algorithm } & \multicolumn{3}{|c|}{ Statistics } \\
\cline { 2 - 5 } & Bias & RMS & Q \\
\hline \hline \multirow{2}{*}{ Eugenio (2000) } & \begin{tabular}{l} 
Day \\
Night \\
\cline { 2 - 5 }
\end{tabular} & 0.148 & 0.547 & 0.566 \\
\cline { 2 - 5 } & Night & 0.204 & 0.500 & 0.539 \\
\hline
\end{tabular}

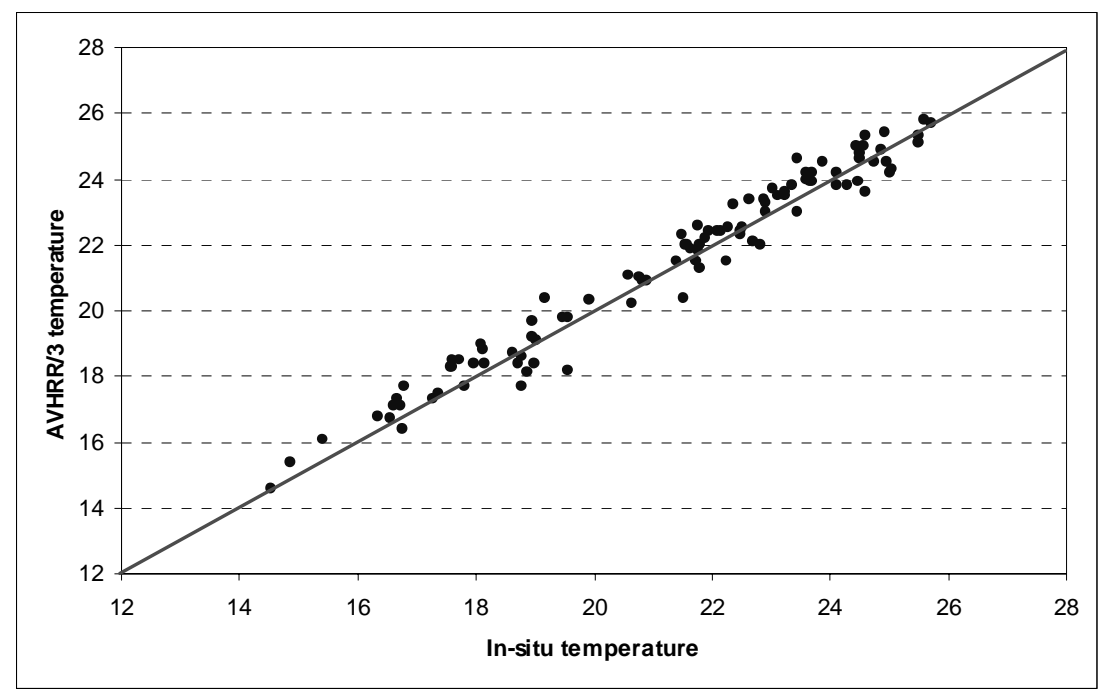

Fig. 4. Comparison between in-situ measurements and AVHRR/3 NOAA 16 SST values.

\section{MODIS ALGORITHM VALIDATION}

With the launch of the Earth Observing System satellite TERRA in December 1999, a new generation of imaging radiometer, the MODIS, was introduced which combines a more extensive suite of spectral bands (36 bands spanning from 0.4-14.4 $\mu \mathrm{m}$ ) and improved instrument technology.

The bands used to measure the SST are in the atmospheric windows at wavelengths of 3.5 to $4.2 \mu \mathrm{m}$ and 10 to $12 \mu \mathrm{m}$, so there are three mid-infrared bands (20, 22 and 23) and two thermal infrared bands (31 and 32). These bands have noise equivalent (NE $\Delta \mathrm{Ts}$ ) around $0.05 \mathrm{~K}$ and a spatial resolution of $1 \mathrm{Km}^{2}$ at the nadir.

The algorithm used to correct the atmosphere contribution using the thermal bands is [1]:

$$
S S T=c_{1}+c_{2} \cdot T_{31}+c_{3} \cdot\left(T_{31}-T_{32}\right)+c_{4} \cdot(\sec (\theta)-1) \cdot\left(T_{31}-T_{32}\right)
$$


where $T_{31}$ and $T_{32}$ are the brightness temperatures measured in bands 31 and 32, $\theta$ is the satellite zenith angle measured at the sea surface $c_{i}$ are the coefficients whose value are determined depending on the difference $T_{31}-T_{32}$.

MODIS provides, as well, the first opportunity to obtain SST using exclusively bands in the medium IR atmospheric window. This window is more transparent and provides the opportunity to derive more accurate SST fields, however this spectral interval suffers from the contamination of the reflected solar radiation in the daytime, limiting its applicability to night-time or where the risk of solar contamination can be confidently discounted. The final form of the algorithm uses channels 22 and 23.

$$
\operatorname{SST} 4=c_{1}+c_{2} \cdot T_{22}+c_{3} \cdot\left(T_{22}-T_{23}\right)
$$

The validation of those algorithms, in our region of interest, was performed using a total of 51 valid match-ups (37 in night-time and the rest during the day) and the results can be summarize at Fig. 5 and Table 3.

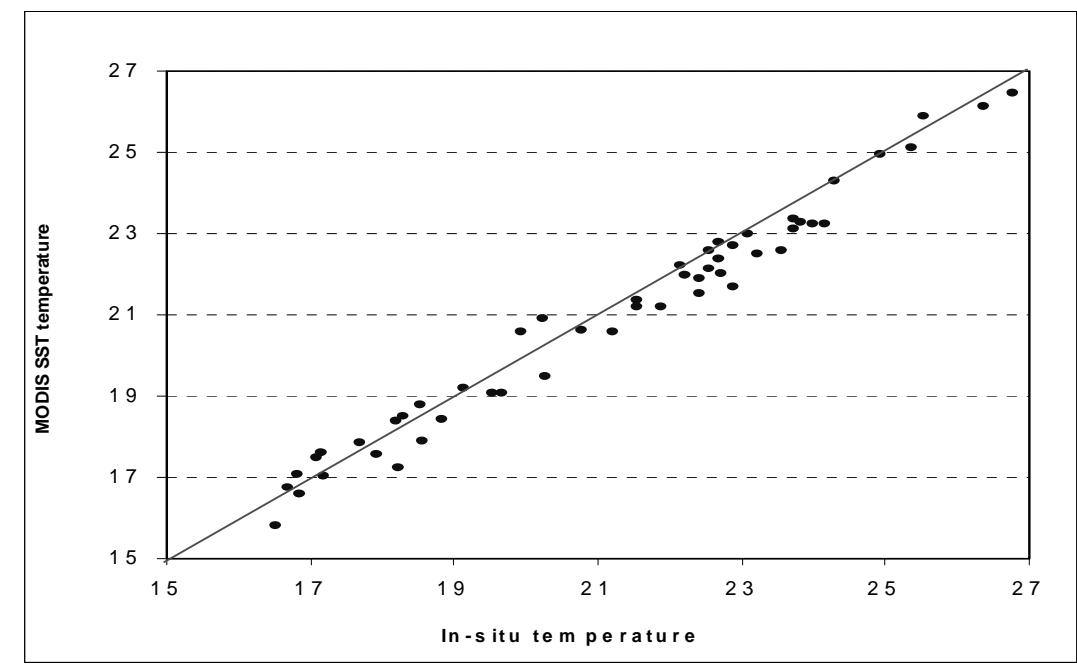

(a)

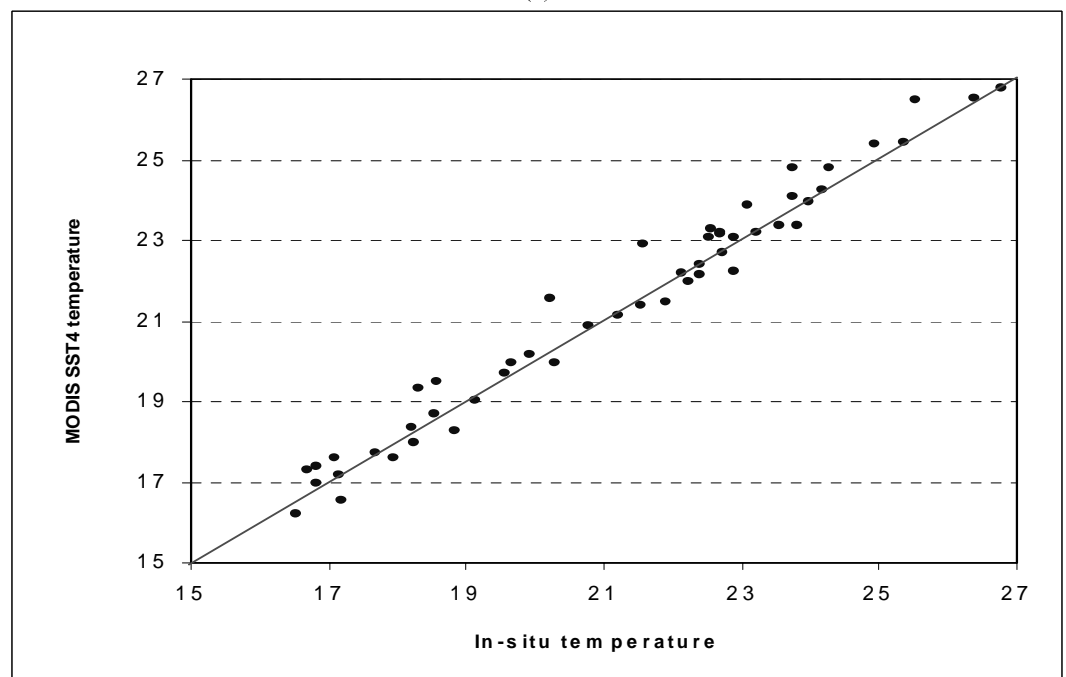

(b)

Fig. 5. Comparison between in-situ measurements and SST values obtained by both IR algorithms.(a)Thermal (SST) and (b) Medium (SST4). 
Table 3. SST Error Statistics applying the MODIS Algorithms to the TERRA-MODIS Images.

\begin{tabular}{|c|l|c|c|c|}
\hline \hline \multirow{2}{*}{ SST estimation algorithm } & \multicolumn{3}{|c|}{ Statistics } \\
\cline { 2 - 5 } & Bias & RMS & Q \\
\hline \hline \multirow{2}{*}{$\begin{array}{c}\text { Thermal Infrared } \\
\text { (SST) }\end{array}$} & $\begin{array}{l}\text { Day } \\
\text { Night }\end{array}$ & -0.251 & 0.505 & 0.563 \\
\hline $\begin{array}{c}\text { Night } \\
\text { Medium Infrared } \\
\text { (SST4) }\end{array}$ & $\begin{array}{l}\text { Day } \\
\text { Night }\end{array}$ & -0.303 & 0.509 & 0.593 \\
\hline Night & 0.019 & 0.521 & 0.561 \\
\hline
\end{tabular}

\section{CONCLUSIONS}

This paper deals with the validation of the AVHRR/3 (NOAA 16) and MODIS (TERRA) algorithm to retrieve the SST within the CANIGO area.

A total number of 1566 in situ temperature measurements were collected from the ARGO project and the Oceanographic Service of the University of Las Palmas Gran Canaria, however after a very stringent filtering based on several restrictions (temporal, spatial, clouds, aerosols, surface warming, quality indicators and standard deviation) only 100 and 51 valid match-ups were considered in the validation database for AVHRR/3 and MODIS respectively.

The performance of the AVHRR/2 operational algorithm used in our Remote Sensing Center was assessed for the AVHRR $/ 3$ and the obtained accuracy for the SST estimation is better than $0.6^{\circ} \mathrm{C}$. With respect to the MODIS sensor, both, thermal and mid-infrared, algorithms achieve residual errors below $0.6^{\circ} \mathrm{C}$.

Finally, daytime match-ups have been excluded from the validation process and the statistics remain basically unchanged except for the MODIS mid-infrared case where the SST estimation achieves accuracies in the order of $0.35^{\circ} \mathrm{C}$. This result is consistent with the well known solar contamination that affects theses spectral bands.

In conclusion we can state that the analysed algorithms provide acceptable precision in the estimation of the sea surface temperature in our oceanographic region of interest, however in the line with the foreseen trends, as specified in the GODAE (Global Ocean Data Assimilation Experiment) [10], the SST must be retrieved with absolute accuracies below $0.3^{\circ} \mathrm{C}$. In consequence at the light of the mentioned results the coefficients will have to be regionally optimized putting special care in the bulk-skin merging conditions.

\section{ACKNOWLEDGMENTS}

This work was supported by the Educational Council of the Canary Islands Government (Contract PI 2002/63). In addition, authors would like to thank the NASA Distributed Active Archive Center for the provision of the MODIS data.

\section{REFERENCES}

1. O. Brown and P. Minnett, (1999) “MODIS Infrared Sea Surface Temperature Algorithm, Algorithm Theoretical Basis Document," Version 2.0 , April 30, 1999.

2. C.J. Donlon, P.J. Minnett, C. Gentemann, T. J Nightingale, I. J. Barton, B. Ward and J. Murray, Towards improved validation of satellite sea surface skin temperature measurements for climate research.J. Climate, 2001. 
3. Argo: http://www.coriolis.eu.org/coriolis/anglais/project/project.htm

4. F. Eugenio, J. Marcello, F. Marqués, A. Hernández-Guerra, and E. Rovaris, "A real time automatic acquisition, processing and distribution system of AVHRR and SeaWiFS imagery," IEEE Geoscience and Remote Sensing Newsletter, 2001.

5. P.W. Saunders and K.T. Kriebel, "An improved method for detecting clear sky and cloudy radiances from AVHRR data." International Journal of Remote Sensing, vol. 9, 127-150, 1988.

6. F. Eugenio and F. Marqués, "Automatic satellite image georeferencing using a contour matching approach," IEEE Trans. Geosci. Remote Sensing, vol 41, n¹2, pp. 2869-2880, 2003.

7. NASA Distributed Active Archive Center Web Page: http://daac.gsfc.nasa.gov/data/dataset/MODIS/

8. C. Coll, V. Caselles, J.A. Sobrino, and E. Valor, "On the atmospheric dependence of split-window equation for land surface temperature." International Journal of Remote Sensing, vol. 15, 105-122, 1994.

9. F. Eugenio, J. Marcello, A. Hernández-Guerra, and E. Rovaris, "Regional optimization of an atmospheric correction algorithm for the retrieval of sea surface temperature from the Canary Islands-Azores- Gibraltar area using NOAA/AVHRR data," International Journal of Remote Sensing, in Press.

10. Global Ocean Data Assimilation Experiment: http:/www.bom.gov.au/GODAE/ 